\title{
Detectability and choice during visual search: Joint effects of sequential priming and discriminability
}

\author{
PATRICIA M. BLOUGH \\ Brown University, Providence, Rhode Island
}

\begin{abstract}
Pigeons searched for symbolic targets among heterogeneous distractor items displayed on a video monitor. Phase 1 varied target identity and overall display size, thus establishing differential discriminabilities of three target symbols. Phase 2 varied the relative probability of these targets within sessions. The findings showed that reaction time was lower not only when targets were more discriminable, but also when they were relatively frequent; these effects did not depend on the discriminability of the less frequent targets. Phase 3 was similar in design but provided occasional choice trials on which two targets appeared. The birds were more likely to respond to the more frequent target on such trials only if it was also the most discriminable. The data are not consistent with certain predictions from Guilford and Dawkins' (1987) reinterpretation of effects attributed to search images. The data indicate that detection and choice are modified jointly by priming-induced expectancies and stimulus-driven perceptual processes.
\end{abstract}

Studies of animal perception have suggested ties between naturally adaptive behavior and rules governing human visual performance. In the case of foraging birds, for example, proficiency improves with the discriminability of the prey (Dawkins, 1971; Gendron, 1986). This outcome replicates findings from numerous studies of visual search in which humans detect targets more quickly or accurately when they are dissimilar to distractor items (e.g., Estes, 1972; McIntyre, Fox, \& Neale, 1970). In work with pigeons, we have shown systematic relationships between target-distractor similarity and search reaction time (D. S. Blough, 1988; D. S. Blough \& P. M. Blough, 1990; P. M. Blough, 1984).

In both naturalistic and laboratory search paradigms, the role of attention has been of special interest. Studies of human search frequently modify attentional focus by operations that provide information about forthcoming targets. Such operations, known as primes, improve search performance and point to the role of expectancy (e.g., P. M. Blough, 1989; Posner \& Snyder, 1975). Posner and Snyder note that the benefits of priming are offset by costs; that is, the attentional focus interferes with detection outside the focal region (see also Eriksen \& Yeh, 1985). It is unlikely that such interference is complete, however. Certain stimulus classes appear to impose fewer attentional demands and thus to bypass areas of focus. For example, visual "features"' (Treisman \& Gelade, 1980) and abrupt onsets (Jonides \& Yantis, 1988) are detected

This research was supported by Grant BNS 88-19876 from the National Science Foundation. I am grateful to Donald S. Blough and Cynthia Langley for their comments. Correspondence concerning this article should be addressed to Patricia M. Blough, Department of Psychology, Brown University, Providence, Rhode Island 02912 (e-mail: patblo@brownvm.bitnet). rapidly and accurately despite the presence of distractors. Yantis and Jonides (1990) distinguish stimulus-driven, "bottom-up" variables from "top-down" factors such as expectancy, and they note instances in which the associated processes seem to compete with each other.

A current issue in the foraging literature seems relevant to this sort of competition. The phenomenon of interest is seen when an abundant food item is taken with a probability that exceeds its relative frequency in a patch. Some accounts suggest that abundancy enhances attentional focus, with attendant costs for the detection of less frequent prey. For example, Tinbergen (1960) proposed that birds formed a search image that highlighted abundant prey but had filter-like properties that could interfere with the capture of alternative food items. Bond (1983) found that pigeons overselected relatively frequent grain types; in his view, rapid captures of a given item induced a specific attentional mode that restricted search to that grain. In recent papers (P. M. Blough, 1989, 1991), I argued that a sequence of encounters with a given target constitutes a priming operation that directs attentional resources to that item. This work did not test directly for interference effects. However, it suggested that sequential priming would not fully block the detection of alternative targets; that is, poorly concealed but unprimed targets were detected more quickly than targets that were highly concealed and primed. Related work (Bond \& Riley, 1991; Reid \& Shettleworth, in press) indicates that sequential priming interferes relatively little with the detection of salient nonprimed items.

An alternative account of the search-image phenomenon rejects attentional models. Instead, it proposes that the effect of abundancy is to modify search strategy (Gendron, 1986; Gendron \& Staddon, 1983; Guilford \& Dawkins, 1987). The argument invokes the relationship, 
mentioned above, between search reaction time and target-distractor discriminability. It notes that a relatively high search rate would be optimal if the patch contained food that was both abundant and conspicuous. However, if the abundant item were cryptic, a reduced rate would optimize capture or reinforcement. The search-rate hypothesis proposes that the foraging animal adjusts its speed according to the concealment of the most frequent item. In their analysis, Guilford and Dawkins distinguished between interference effects implied by attentional and search-rate models. Although attentional accounts imply reduced detectability of any nonabundant prey, the searchrate hypothesis predicts an asymmetric form of selectivity: Detection of infrequent targets would depend only on their discriminability relative to the abundant item that controls speed. Thus, a low search rate, adopted for cryptic items, would not interfere with the detection of more salient targets. However, an animal searching quickly for conspicuous prey would be likely to miss concealed targets, which require more viewing time. As noted above, laboratory data (P. M. Blough, 1989, 1991; Bond \& Riley, 1991) suggested some asymmetry, but not always to the extent required by Guilford and Dawkins' analysis.

Asymmetric interference effects, although predicted by search-rate accounts, are not necessarily inconsistent with attentional explanations of search-image phenomena. As noted above, stimulus factors also modify attention and may compete with processes induced by priming (Yantis \& Jonides, 1990). It seems reasonable to speculate that more salient targets are stronger competitors for attentional resources. However, attentional accounts would predict less extreme asymmetries than those outlined by Guilford and Dawkins (1987), who state that abundancy should have no effects on relatively salient targets. Yantis and Jonides' competing view suggests a graded relationship in which top-down and bottom-up processes modify each other according to their relative strengths.

The present study addressed the selectivity of sequential priming. In particular, it considered competition between priming-induced and stimulus-driven processes during pigeon visual search. The experimental arrangements used artificial, computer-generated stimuli but were designed to mimic sequences that might occur during foraging. Targets varied in discriminability. Expectancy was manipulated by changing the probability of a given target. Phase 1 established discriminability relations among the targets. Phase 2 assessed the joint effects of target discriminability and probability on detection accuracy and reaction time (RT). Phase 3 extended studies of sequential priming to choice; it considered the extent to which this measure reflected the frequency and probability effects seen in Phase 2.

\section{METHOD}

\section{Subjects}

The subjects were 6 White Carneaux pigeons. Their previous laboratory experience was limited to a variety of brief keypecking tasks associated with an undergraduate course. Each bird's weight was maintained at a level approximating $80 \%$ of its free-feeding value; small downward adjustments were made for the subjects if they showed long pauses during training.

\section{Apparatus}

Three experimental boxes each enclosed a $29 \mathrm{~cm}$ wide $\times 31 \mathrm{~cm}$ deep $\times 36 \mathrm{~cm}$ high subject chamber. An opening in the chamber's front panel, centered $24 \mathrm{~cm}$ above the floor, admitted a monochromatic video monitor mounted just behind a $10.5 \mathrm{~cm}$ wide $\times 6 \mathrm{~cm}$ high $\times 1.2 \mathrm{~cm}$ deep frame. Embedded within the frame were six pairs of photodetector units. Programming software used the input from these units to separate the monitor screen into six equally wide vertical segments for the purpose of localizing keypecks. Below the frame was a smaller opening that contained a dim lamp and provided access to a food tray. A second dim lamp, shielded from below, was mounted centrally above the video monitor. Each chamber was equipped with a loudspeaker that delivered white masking noise. Boxes were interfaced with Atari computers located in an adjacent room; the computers sensed responses and controlled experimental events. (For further details, see D. S. Blough, 1986.)

\section{Procedure}

The birds were run in two groups, Group 1 (Birds 478, 463, and 468) and Group 2 (Birds 90,492 , and 495). Differences in session lengths, specified below, were necessitated by computer availability during the time periods assigned to these groups.

Stimulus displays. Test displays consisted of black symbols on a light background. The symbols subtended approximately $2^{\circ}$ of arc and were separated by a minimum of $5^{\circ}$. Symbols could appear in any of 36 preassigned screen locations, arranged in irregular rows and columns; 6 locations were assigned to each of the 6 response segments. Each display included one or two targets and a variable number of distractors. The target set consisted of a filled heart (HRT), a backslash symbol (SL), and an uppercase B. On a given trial, the target could appear in any of the 36 screen locations. Its placement was determined by a random-block method that assigned it equally often to each response segment during a session. A set of distractor items was made up of 36 nontarget symbols consisting of letters, numerals, and other standard characters. On a given trial, the designated number of distractors were drawn at random and without replacement from this set and assigned at random to the remaining screen locations. Target and distractor assignments were redetermined on each trial.

Response contingencies. A response was defined as an uninterrupted series of five pecks to a given screen segment. Following a response to the target segment, the display screen turned white for a minimum of $1.0 \mathrm{sec}$; with a given probability and duration, mixed-grain reinforcement became available, and the screen remained light during this period. Reinforcement probability and duration were adjusted so that each bird maintained its running weight during experimental sessions; after training, probability values were fixed for each bird, ranging from .06 to .10 . Durations could vary from session to session, depending on the bird's initial weight; however, the change between sessions never exceeded $.5 \mathrm{sec}$. Following reinforcement, the screen turned medium gray and a $5.5-\mathrm{sec}$ intertrial interval (ITI) began. At the onset of a new trial, the screen turned light gray and the test display appeared. Following a response to a nontarget segment (error), the ITI began and the trial was rerun until a correct response occurred. Such rerun trials were not included in the data or in trial counts.

Experimental conditions. The experiment consisted of three phases, which established target discriminability (Phase 1) and evaluated effects of discriminability and target probability on search performance (Phase 2) and choice (Phase 3).

Phase 1 began when training was complete (see below) and assessed the effect of display size for each of the three targets. Daily sessions used a single target symbol, and sessions associated with 
each of the three targets rotated. Display size varied within sessions $(12,24$, or 36 items); size on a given trial was selected by a random-block procedure so that each of these conditions occurred equally often. ITIs lasted $5.5 \mathrm{sec}$. Sessions consisted of 600 trials for both groups. Final data were based on the first four sessions that met a stability criterion for trend (P. M. Blough, 1984).

Phase 2 assessed the effects on RT of target frequency and discriminability. Display size was constant at 36 items. Each session included frequent trials with a single main target and infrequent probe trials on which one of the alternate targets appeared. The identity of the main target rotated among sessions. The rule governing trial sequences was as follows: The main target appeared during a sequence of 15 trials. On the 16 th trial, a probe could appear with a probability of 0.20 . If the probe was not selected, the same decision was made on the following trial; this procedure continued until either a probe was selected or a sequence of 19 trials was completed. In the latter case, the probe target appeared on the 20th trial. After a probe trial, a new sequence began. ITIs lasted $5.5 \mathrm{sec}$.

The outcome of Phase 1 modified the target sets used in this phase and in Phase 3. Birds 478, 463, 468 (Group 1) and Bird 495 (Group 2) showed clear differences in discriminability among the HRT, SL, and B target symbols. Thus, these subjects experienced sessions in which either of these symbols could be the main target; the three session types rotated in the same order for these birds. During sessions associated with a given main target, probe targets could be either of the alternate target symbols, which appeared equally often. Birds 90 and 429 (Group 2) did not show discriminability differences between the SL and the $\mathrm{B}$ targets and did not experience the B target in this phase or in Phase 3. The main target could be the HRT or the SL in alternating sessions, and all probe trials presented the alternate target symbol. For Group 1, sessions were made up of 900 trials, and there were 8 sessions for each main target. For Group 2, sessions were made up of 600 trials, and there were 12 sessions for each main target.

Phase 3 was similar in plan to Phase 2 . However, probe trials presented two targets in separate and randomly selected screen segments. One member of the pair was the main target for the session; the second was an alternate target. Since Birds 478, 463, 468,

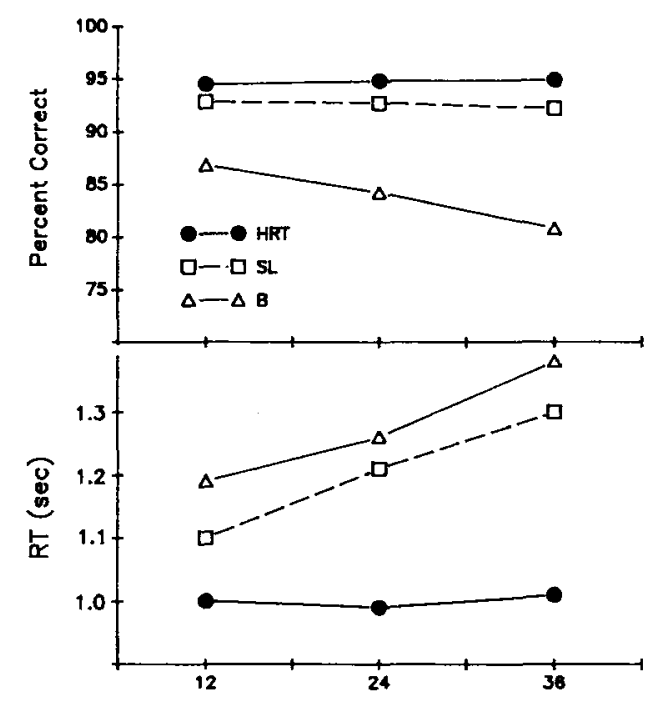

Figure 1. Accuracy (top panel) and reaction time (RT) (bottom panel) as a function of display size. Data are from Phase 1. The different targets were presented in separate sessions. Points are means of within-session means (accuracy data) or means of within-session medians (RT data). and 495 experienced three target symbols altogether, there were two types of probe trial in each session; for Birds 90 and 429, there was a single type of probe trial. On the trials just preceding a probe, the display contained two main targets. The recorded choice was a sequence of five pecks at one of the targets.

For Group 1, sessions consisted of 800 trials, and there were 10 such sessions altogether for each of the three main targets. For Birds 90 and 429 (Group 2), sessions consisted of 600 trials, and there were 12 sessions for each of the two main targets. Bird 495 also experienced 600 -trial sessions; however, there were 13 sessions for each of the three main targets in order to obtain a comparable number of presentations of each probe pair.

Training and final data collection. All birds learned to peck at target letters through autoshaping and, in some cases, handshaping methods. Subsequent training gradually reduced the probability of food reinforcement and added increasing numbers of distractors; training continued until the bird's accuracy stabilized at a display size of 36 items. In individual training sessions, a single target was used; its identity initially rotated between sessions, but it was necessary to extend the number of sessions with the SL and the B targets. As noted above, a stability criterion determined the sessions to be included in the final data for Phase 1 . Subsequent phases began with a single practice session for each main target; data from these did not contribute to the total session count or to the final data set. Every session began with 12 warmup trials that displayed only the target and that were not counted.

\section{RESULTS}

In the following analyses, RT refers to the interval between display onset and the first peck of a sequence that defined a correct response. Summary values are means of within-session medians. Accuracies refer to mean percent correct responses averaged over sessions.

\section{Phase 1}

Phase 1 explored the discriminabilities of the three target symbols. Figure 1 summarizes the findings. The top panel shows accuracy as a function of display size for the three targets; the separation among these functions was present in the data of the individual subjects, with a single exception: Bird 495 showed similar accuracies to the HRT and SL symbols. The results were submitted to an analysis of variance (ANOVA) for within-subject designs. Both main effects and their interaction were significant $(p<.01)$; for target identity, $F(2,10)=27.5$; for display size, $F(2,10)=7.5$; for the interaction, $F(4,20)=7.2$.

The bottom panel of Figure 1 summarizes display-size functions for the RT measure. An ANOVA showed significant effects $(p<.001)$ of both main factors and their interaction; for target identity, $F(2,10)=24.2$; for display size, $F(2,10)=39.6$; for the interaction, $F(4,20)=7.83$.

Figure 2 shows individual display-size functions for the RT measure. For 4 birds, there was clear separation among the three functions. For Birds 90 and 429, however, RTs associated with the SL and the B symbols were not consistently different. Thus, for these 2 subjects, only the HRT and SL symbols were used in subsequent phases.

Overall, Phase 1 established differential discriminabilities for the three targets. The significant interactions 

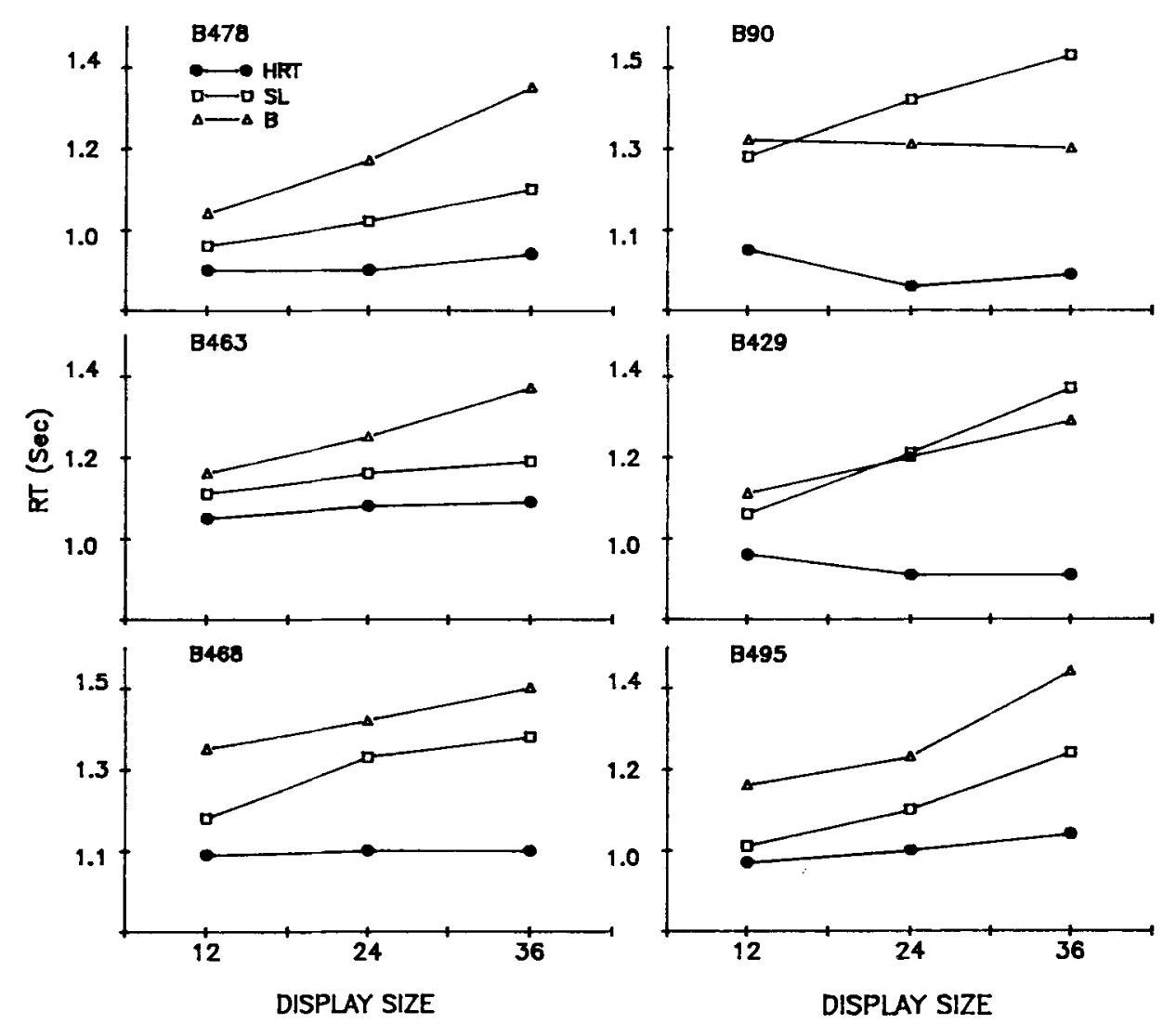

Figure 2. Individual reaction time (RT) data from Phase 1. Data are plotted as in Figure 1, but separate panels show functions for each of the 6 subjects.

indicate that these differences are accentuated by large displays.

\section{Phase 2}

Phase 2 evaluated joint effects of target frequency and discriminability on detection. Figure 3 summarizes accuracy (numerals) and RT (bars) for Phase 2. Individual panels are organized to highlight frequency effects within particular target combinations. For example, the left panel compares responses to the HRT and the SL targets. The bars labeled MT show RTs to the HRT during sessions in which the HRT was the main target (open bar) or to the SL during sessions in which the SL was the main target (striped bar). The open bar labeled Pr shows responses to the HRT when it appeared as an infrequent probe during sessions in which the SL was the main target; the striped bar labeled Pr shows responses to the SL during sessions when the HRT was the MT. Thus, within each panel, frequency effects are seen in comparisons within a bar pair. Discriminability effects are seen in comparisons between bar pairs. The means shown here indicate that RTs were lower when targets were more probable as well as when they were more discriminable.

Figure 3 also shows accuracy as percent correct figures appearing above the corresponding RT bar. These means indicate enhanced accuracy associated with more frequent, as well as with more discriminable, targets.
Data from the separate panels in Figure 3 were submitted to separate ANOVAS, details of which appear in Table 1, which has an organization similar to Figure 3. A comparison of responses to the HRT and SL targets (top segment of Table 1) showed that the effects of target discriminability were significant by both accuracy and RT measures. Target frequency significantly affected RT but did not significantly affect accuracy. A comparison of responses to the HRT and B targets (center segment of Table 1) showed that the effect of discriminability was significant for both the accuracy and RT measures but that frequency had no significant effect on either measure. A comparison of responses to the SL and B targets (bottom segment of Table 1) showed that both discriminability and frequency effects were significant for the accuracy measure. Frequency also significantly affected RT, and discriminability approached significance for this measure. None of these data showed significant interactions.

Overall, Phase 2 data indicated that speed of target detection was affected by its within-session probability as well as by its similarity to its background. Accuracy was less sensitive to frequency effects.

\section{Phase 3}

Phase 3 explored choice between simultaneously presented targets that differed in discriminability and proba- 


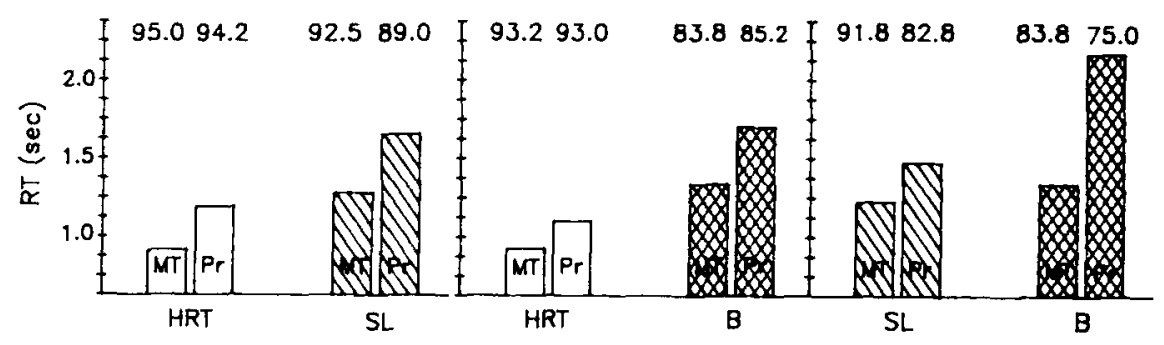

Figure 3. Accuracy and reaction time (RT) as a function of target identity and probability. Data are from Phase 2. Bars show RTs, and numerals above show accuracy for the corresponding condition. Patterns on bars designate target identity, which is also shown below each bar pair. Highfrequency targets (MT) appeared in long sequences; low-frequency targets (Pr) appeared during a single trial at the end of a sequence.

Table 1

ANOVA Evaluation of Discriminability and Frequency Effects on RT

\begin{tabular}{|c|c|c|c|c|c|c|c|c|c|c|c|c|}
\hline \multirow[b]{3}{*}{ Comparison } & \multicolumn{6}{|c|}{ Accuracy } & \multicolumn{6}{|c|}{$\mathbf{R T}$} \\
\hline & \multicolumn{3}{|c|}{ Discriminability } & \multicolumn{3}{|c|}{ Frequency } & \multicolumn{3}{|c|}{ Discriminability } & \multicolumn{3}{|c|}{ Frequency } \\
\hline & $F$ & $d f$ & $p$ & $F$ & $d f$ & $p$ & $F$ & $d f$ & $p$ & $F$ & $d f$ & $p$ \\
\hline HRT-SL & 7.6 & 1,5 & .04 & 2.3 & 1,5 & $>.1$ & 17.6 & 1,5 & .008 & 11.7 & 1,5 & .02 \\
\hline HRT-B & 27.7 & 1,3 & .01 & .1 & 1,3 & $>.1$ & 21.3 & 1,3 & .02 & 3.7 & 1,3 & $>.1$ \\
\hline SL-B & 12.3 & 1,3 & .04 & 36.8 & 1,3 & .01 & 6.2 & 1,3 & .09 & 12.1 & 1,3 & .04 \\
\hline
\end{tabular}

Note-Table 1 shows target pairs submitted to each ANOVA comparison, outcomes for both accuracy and RT measures, and statistical values associated with discriminability and frequency factors evaluated. Rows correspond to individual panels in Figure 3. There were no significant interactions.

bility. Table 2 summarizes those data. Pairs of rows refer to sessions having the indicated main targets; individual rows show individual choice pairs. For example, the top segment shows data for trials during sessions in which the HRT was the main target. The first row shows outcomes of choice trials that presented the HRT and the SL; the second row shows outcomes of choice trials that presented the HRT and the B. Within-subject $t$ tests evaluated the hypothesis that choice was random $(50 \%)$. The hypothesis was rejected $(p<.01)$ in all cases in which the most frequent target was also the more discriminable [denoted by asterisks in Table $2 ; t(5)=4.70$ when the HRT appeared with a SL probe, $t(3)=8.98$ when the HRT appeared with a B probe, and $t(3)=8.01$ when the SL appeared with the B probe]. When the main target was the less discriminable symbol, the findings were more variable. When the SL appeared with the HRT probe, choice did not depart significantly from chance, and this was also the case when the B appeared with the SL probe. However, when the B appeared with the HRT probe, the HRT was chosen more frequently $[t(3)=3.44$, $p<.04]$. Thus, target probability modified the tendency to choose the more discriminable target, although less discriminable targets never dominated choice.

Table 3 summarizes RTs obtained during this phase. Rows are organized by main target and choice pair, as they were in Table 2. The second column shows data from the bulk of the trials, when a single target was presented. The discriminability effect persisted; that is, the pattern of RTs conformed to that seen in Phases 1 and 2. The fourth column shows RTs associated with main target choices during two-target probe trials. RTs were shorter for a given target when it appeared in a choice display than when it appeared alone (compare 2nd and 4th columns). Within-subject $t$ tests established the reliability of this difference in all but one case: for the HRT (1st and 2 nd rows), $t(5)=3.42$ and $t(3)=5.43$, respectively, $p<.02$ in both cases; for the SL (3rd and 4th rows), $t(5)=3.68, p<.02$, and $t(3)=1.91, .1>p>.05$; for the $B$ (5th and 6th rows), $t(3)=6.79$ and 6.87 , respectively, $p<.01$ in both cases. The fifth column in Table 3 shows RTs associated with the less frequent target when it was chosen during probe trials. ANOVAS such as those applied to Phase 2 data evaluated the effects of target discriminability and frequency on choice RTs. Discriminability was significant only in the comparison of the SL and the B targets $[F(1,3)=11.8, p=.04]$; this factor approached significance in the comparison of the HRT and the SL targets $[F(1,5)=4.9, .1>p>.05]$. There were no significant effects of target frequency, and no interaction between the two factors.

\section{DISCUSSION}

During search for multiple targets, both target discriminability and probability affected performance. Reaction time was the most sensitive measure of these effects, but many of the findings were also reflected by accuracy 
Table 2

Choice Data (Phase 3)

\begin{tabular}{ccc}
\hline Main Target & Choice Pair & Choice 1 \\
\hline HRT & HRT-SL & $75.8^{*}$ \\
& HRT-B & $84.2^{*}$ \\
SL & SL-HRT & 55.7 \\
& SL-B & $81.8^{*}$ \\
B & B-HRT & $31.2^{*}$ \\
& B-SL & 56.2
\end{tabular}

Note-Choice 1 refers to the percentage of trials on which the birds responded to the first item in the choice pair. * Denotes proportions that differed significantly from chance $(50 \%)$. Percentages do not include trials on which both targets were missed; misses occurred on $0.4 \%$ to $10.7 \%$ of the trials and did not exceed $5 \%$ for most of the birds.

scores. Conditions that modified detectability also affected choice; in particular, for simultaneously displayed targets, choice of a given item exceeded chance when it was more discriminable and more probable within the session. Discriminability overrode frequency in one case: the highly concealed (B) was less likely to be chosen than the salient, but infrequent, alternative (HRT).

Recent, more naturalistic, work of Bond and Riley (1991) and of Reid and Shettleworth (in press) has also examined the role of target frequency and discriminability. Both studies identified conditions in which single-target sequences interfered with performance on subsequent trials with an alternative item. In a paradigm conceptually similar to that of the present Phase 2, Bond and Riley did not find impaired detection of a conspicuous target following a run with a cryptic item. With a like procedure, Reid and Shettleworth (Experiment 2) compared targets of equal discriminability and did not find frequency effects. In their Experiment 3, these authors turned to a choice procedure similar to that of the present Phase 3. In this case, same-target sequences favored choice of that target. Overall, the discriminability effects are clear in all three studies, but the frequency effect was more robust in the present experiment, which used exceptionally large frequency differences.

It is tempting to account for the target frequency effect in terms of differential reinforcement; that is, there were more reinforcers per session associated with the main targets than with the probes. However, recent results from this laboratory (D. S. Blough, 1992) indicate that differential reinforcement probability affects RT but that this measure is not sensitive to relative reinforcement frequency. In the present experiments, reinforcement probability was constant. Furthermore, the between-session target-probability effect is conceptually similar to those seen in within-session designs that equalized overall reinforcers per target (P. M. Blough, 1989, 1991; Bond \& Riley, 1991; Pietrewicz \& Kamil, 1979, 1981).

The findings of this study address the relationship between stimulus factors and priming-induced expectancies in guiding attention. In most comparisons, both of these variables affected detectability and choice. The outcome is not consistent with Guilford and Dawkins' (1987) anal- ysis, which predicts that abundancy will interfere with the detection of less frequent alternatives only when those alternatives are relatively concealed. Indeed, there was no evidence for a dependent relationship between target frequency and discriminability. Because stimulus effects also contributed to detectability, the data took on a pattern that resembled in direction, if not in degree, the one predicted by Guilford and Dawkins. For example, the infrequent but salient targets tended to be detected as well as or more quickly than frequent but less discriminable items (Figure 3). The overall outcome is consistent with attentional accounts that consider joint effects of bottom-up and top-down factors (Bundesen, 1990; Yantis \& Jonides, 1990). It appears that stimulus-driven effects are not limited to a special class of attention-capturing events (Jonides \& Yantis, 1988; Treisman \& Gelade, 1980) but contribute in a graded fashion according to their salience.

The findings did not show significant frequency effects in HRT-B comparisons either for detectability (Figure 3) or choice (Table 2), although differences comparable to other comparisons were present. Quite possibly, a Type II statistical error occurred. Alternatively, one might speculate that the strong bottom-up influence exerted by the HRT overrode the top-down mechanisms tuned to the poorly discriminable B. In the HRT-SL comparison, in which a frequency effect did occur, the moderately salient SL would have competed more successfully with the HRT.

The third phase of this study extended work on detectability to choice. It confirmed the expected relationship, showing that targets seen more quickly are more frequently selected. This outcome is consistent with extensions to natural foraging, which assume an association between RT and prey selection. One approach to the nature of this association could start with the Vickers (1970) model of attention, applied by Bond \& Riley (1991) to their analysis of searching images. Generally, this model proposes that RTs reflect the time engaged in sampling information within a given display. Decisions based on these samples accumulate toward criteria, which are mapped individually to available responses. When a criterion is met, the associated response occurs. In singletarget trials, correct RTs reflect the accumulation time associated with the appropriate criterion. In choice trials,

Table 3

RTs for Single-Target and Choice Displays (Phase 3)

\begin{tabular}{lcclcc}
\hline Target & $n$ & Alone & Choice Pair & Choice 1 & Choice 2 \\
\hline HRT & 6 & 1.08 & HRT-SL & 1.02 & 1.13 \\
& 4 & 1.09 & HRT-B & 1.05 & 1.07 \\
SL & 6 & 1.28 & SL-HRT & 1.12 & 1.10 \\
& 4 & 1.25 & SL-B & 1.21 & 1.44 \\
B & 4 & 1.34 & B-HRT & 1.13 & 1.08 \\
& 4 & 1.34 & B-SL & 1.20 & 1.18 \\
\hline
\end{tabular}

Note-RTs are in seconds. Choice 1 refers to the first target in the choice pair; Choice 2 refers to the second target. For a given target, RTs during single-target trials (alone) differ slightly according to choice pair because fewer birds $(n)$ experienced conditions using the B target. 
there are two correct responses, and the chances are greater that one of the associated criteria will be met in a given amount of time. As can be seen in Table 3, RTs in two-target displays were indeed shorter than those for single targets.

In "race models" such as Vickers', RTs are longer for less detectable targets because the sampling process is more extended. Typically, however, the frequency distributions for the different targets overlap (illustrated in D. S. Blough, 1988, Figures 15 and 16). In two-target displays, the more detectable target is selected more frequently because the associated criterion is passed first on the greater proportion of trials. Nonetheless, because of the overlapping RT distributions, there are trials on which the less detectable target is seen first and thus selected. This outcome is consistent with choice allocation, as shown in Table 2. What is more interesting is that this analysis suggests that RTs associated with different targets should be more similar on choice trials than they are when the targets are presented singly. This prediction emerges from the race logic; $\mathrm{RT}$ reflects the time required for one or the other criterion to be passed. Trials on which the less detectable target is selected are those on which the associated criterion happens to be passed first; thus, RTs for such trials weight more heavily the shorter end of the distribution associated with this target. Comparisons between choice RTs and comparable single-target RTs (Figure 2) are consistent with this prediction. Although there were significant RT differences between discriminable frequent targets and concealed infrequent targets when the targets were presented singly (Figure 2), these differences were diminished and were usually not significant when the pairs were presented simultaneously (Table 3). Specifically, the mean RT difference between the frequent HRT and the SL probe was $.59 \mathrm{sec}$ during single presentations and $.11 \mathrm{sec}$ during simultaneous presentations. For the frequent HRT and the B probe, the respective differences were .63 and $.04 \mathrm{sec}$; for the frequent SL and the B probe, the respective differences were .75 and $.23 \mathrm{sec}$.

This report shares with several others (P. M. Blough, 1989, 1991; Bond \& Riley, 1991; Pietrewicz \& Kamil, 1979, 1981; Reid \& Shettleworth, in press) an attempt to relate data from trialwise procedures to issues relevant to natural foraging. It is important to exercise caution in making such extensions. For example, the present trialwise procedure may not assess natural search rates, since the foraging pigeon can move on to a new area without responding to a target (Reid \& Shettleworth). Furthermore, in the present design, the onset and offset of searching opportunities were strongly marked by intertrial intervals; consequently, errors were penalized by timeouts. Thus, contingencies influencing responding in these situations must differ from those affecting the freeforaging bird. Endler (1991) argued that search rate would be more appropriately measured in paradigms that incorporate the time between target encounters. Nonetheless, it is notable that the abundancy effects reported by Bond
(1983) and replicated by Reid and Shettleworth's Experiment 1 used free-feeding paradigms. Further work should address possible dissociations between the components of behavior that make up search rate.

\section{REFERENCES}

Blough, D. S. (1986). Odd-item search by pigeons: Method, instrumentation, and uses. Behavior Research Methods, Instruments, \& Computers, 18, 413-419.

BLOUGH, D. S. (1988). Quantitative relations between visual search speed and target-distractor similarity. Perception \& Psychophysics. 43, 57-71.

Blough, D. S. (1992). Effects of stimulus frequency and reinforcement variables on reaction time. Journal of the Experimental Analysis of Behavior, 57, 47-50.

Blough, D. S., BLough, P. M. (1990). Reaction time assessments of visual perception in pigeons. In W. C. Stebbins \& M. A. Berkley (Eds.), Comparative perception: Vol II. Complex signals (pp. 245276). New York: Wiley.

Blough, P. M. (1984). Visual search in pigeons: Effects of memory set size and display variables. Perception \& Psychophysics, 35, 344-352.

Blough, P. M. (1989). Attentional priming and visual search in pigeons. Joumal of Experimental Psychology: Animal Behavior Processes, 15, 358-365.

BLough, P. M. (1991). Selective attention and search images in pigeons. Journal of Experimental Psychology: Animal Behavior Processes, 17, 292-298.

BoND, A. B. (1983). Visual search and selection of natural stimuli in the pigeon: The attention threshold hypothesis. Joumal of Experimental Psychology: Animal Behavior Processes, 9, 292-306.

Bond, A. B., RILEY, D. A. (1991). Searching image in the pigeons: A test of three hypothetical mechanisms. Ethology, 87, 203-224.

Bundesen, C. (1990). A theory of visual attention. Psychological Review, 97, 523-547.

Dawkins, M. (1971). Perceptual changes in chicks: Another look at the "search image" concept. Animal Behaviour, 19, 566-574.

ENDLER, J. A. (1991). Interactions between predators and prey. In J. R. Krebs \& N. B. Davies (Eds.), Behavioral Ecology (3rd ed., pp. 169. 196). London: Blackwell Scientific.

ERIKSEN, C. W., YEH, Y. Y. (1985). Allocation of attention in the visual field. Journal of Experimental Psychology: Human Perception \& Performance, 11, 583-597.

EsTes, W. K. (1972). Interactions of signal and background variables in visual processing. Perception \& Psychophysics, 12, 278-286.

Gendron, R. P. (1986). Searching for cryptic prey: Evidence for optimal search rates and the formation of search images in quail. Animal Behaviour, 34, 898-912.

Gendron, R. P., StadDon, J. E. R. (1983). Searching for cryptic prey: The effect of search rate. American Naturalist, 121, 172-186.

Guilford, T., DAwkINs, M. S. (1987). Search images not proven: A reappraisal of recent evidence. Animal Behaviour, 35, 1838-1845.

JoNIDES, J., \& YANTIS, S. (1988). Uniqueness of abrupt visual onset in capturing attention. Perception \& Psychophysics, 43, 346-354.

McIntYre, C., FoX, R., Neale, J. (1970). Effects of noise similarity and redundancy on the information processed from brief visual displays. Perception \& Psychophysics, 7, 328-332.

Pietrewicz, A. T., KAMIL, A. C. (1979). Search image formation in the Blue Jay (Cyanocitza cristata). Science, 22, 1332-1333.

Pietrewicz, A. T., KAMil, A. C. (1981). Search images and the detection of cryptic prey: An operant approach. In A. C. Kamil \& T. D. Sargent (Eds.), Foraging behavior: Ecological, ethological, and psychological approaches (pp. 311-331). New York: Garland STPM Press.

Posner, M. I., SNyder, C. R. R. (1975). Facilitation and inhibition in the processing of signals. In P. M. Rabbitt \& S. Domic (Eds.), Attention and performance, (Vol. 5, pp. 669-682). San Diego: Academic Press. 
Reid, P. J., \& Shettleworth, S. J. (in press). Detection of cryptic prey: Search image or search rate? Journal of Experimental Psychology: Animal Behavior Processes.

Tinbergen, L. (1960). The natural control of insects in pinewoods: I. Factors influencing the density of predation by songbirds. Archives Neerlandaises de Zoologie (Leiden), 13, 256-343.

Treisman, A. M., \& Gelade, G. (1980). A feature-integration theory of attention. Cognitive Psychology, 12, 97-136.
VICKERS, D. (1970). Evidence for an accumulator model of psychophysical discrimination. Ergonomics, 13, 37-58.

YANTIS, S., \& JoNIDES, J. (1990). Abrupt visual onsets and selective attention: Voluntary versus automatic allocation. Joumal of Experimental Psychology: Human Perception \& Performance, 16, 121-134.

(Manuscript received August 19, 1991; revision accepted for publication February 15, 1992.)

\section{Forthcoming Articles}

The following is a list of forthcoming Animal Learning \& Behavior articles that are currently in press. They are given in approximate order of acceptance. Each entry includes the name and address of the author with whom to communicate for further prepublication information.

"Incentive learning and the motivational control of instrumental performance by thirst"' by M. Lopez, B. Balleine, \& A. Dickinson (A.D., Dept. of Experimental Psychology, Univ. of Cambridge, Downing Street, Cambridge CB2 3EB, U.K.)

"The role of predictability in preventing escape deficits following loss of control over food acquisition" by A. Sonoda \& H. Hirai (A.S., Dept. of Psychology, Sophia Univ., 7-1, Kioicho, Choyoda-ku, Tokyo 102, Japan)

"Common coding in pigeons: Partial versus total reversals of one-to-many conditional discriminations"' by T.R. Zentall, L.M. Sherburne, J.N. Steirn, C.K. Randall, K.L. Roper, \& P.J. Urcuioli (T.R.Z., Dept. of Psychology, Univ. of Kentucky, Lexington, KY 40506)

"Pavlovian conditioning in multiple contexts: Competition between contexts for comparator status" by N.J. Grahame, R.C. Barnet, \& R.R. Miller (R.R.M., Dept. of Psychology, SUNY-Binghamton, Binghamton, NY 13902-6000)

"Latent habituation of the orienting response in the preweanling rat'" by R. Richardson \& B.A. Campbell (R.R., Dept. of Psychology, Green Hall, Princeton, NJ 08544-1010)

"Conditioned inhibition of analgesia"' by E.P. Wiertelak, L.R. Watkins, \& S.F. Maier (E.P.W., Dept. of Psychology, Univ. of Colorado, Boulder, CO 80309)

"The influence of spatial irregularity upon radial-maze performance in the rat"' by M.-C. Grobety \& F. Schenk (F.S., Institut de Physiologie Humaine, Rue du Bugnon 7, CH 1005 Lausanne, Switzerland)

"Relative preferences for various bi-valued ratio schedules" by W. Ahearn, P.N. Hineline, \& F.G. David (W.A., Dept. of Psychology, Temple Univ., Philadelphia, PA 19123)

"Conditioning copulatory behavior to an artificial object: Efficacy of stimulus fading'" by M. Domjan, M. McDonald, \& K. Holloway (M.D., Dept. of Psychology, Univ. of Texas, Austin, TX 78712)

"Rats remember not wisely but too well" by S. Adams-Pepper, S. Gagnon, S. Fore, \& H.L. Roitblat (H.L.R., Dept. of
Psychology, Univ. of Hawaii, 2430 Campus Rd., Honolulu, HI 96822)

"Spontaneous recovery in cross-motivational transfer (counterconditioning)'" by M.E. Bouton \& C.A. Peck (M.E.B., Dept. of Psychology, Univ. of Vermont, Burlington, VT 05405)

"Stimulus preference and the transitivity of preference" by T.W. Belke (Dept. of Psychology, Harvard Univ., 33 Kirkland St., Cambridge, MA 02138)

"Effects of the inclination of context lines on perception of the Ponzo illusion by pigeons"' by K. Fujita, D.S. Blough, \& P.M. Blough (K.F., Dept. of Psychology, Primate Research Institute, Kyoto Univ., Inuyama, Aichi 484, Japan)

"Age differences in the acquisition and retention of concurrent discriminations by monkeys" by L. Bakner \& F.R. Treichler (F.R.T., Dept. of Psychology, Kent State Univ., Kent, OH 44242)

"Delayed matching-to-successive-samples in pigeons: Shortterm memory for item and order information' 'by S.E. MacDonald (Dept. of Psychology, York Univ., Atkinson College, 4700 Keele St., Toronto, ON, Canada M3J 1P3)

"Transfer of contextual control across similarly trained conditioned stimuli" by D. Swartzentruber (Dept. of Psychology, Univ. of Pennsylvania, 3815 Walnut St., Philadelphia, PA 19104)

"Social transmission of food preferences among Norway rats by marking of food sites and by gustatory contact' ' by K.N. Laland \& H.C. Plotkin (K.N.L., Dept. of Psychology, Univ. College London, Gower Street, London WC1E 6BT, U.K.)

"Coherence of retrieval cues, rather than additivity, determines prior cuing effectiveness in the rat" by $P$. Gisquet-Verrier (Lab. de Neurobiologie de l'Apprentissage et de la Mémoire, URA 1491, C.N.R.S., 91198 Gif sur Yvette Cedex, France)

"Sexual approach conditioning: Omission contingency tests" by L.L. Crawford \& M. Domjan (M.D., Dept. of Psychology, Univ. of Texas, Austin, TX 78712) 